\title{
An atypical biceps brachii and coracobrachialis muscles associated with multiple neurovascular aberrations: a case report with clinical significance
}

\author{
M. Piagkou1 ${ }^{1}$, T. Totlis², N. Anastasopoulos², N. Lazaridis², K. Natsis² \\ ${ }^{1}$ Department of Anatomy, School of Medicine, Faculty of Health Sciences, National and Kapodistrian \\ University of Athens, Greece \\ 2Department of Anatomy and Surgical Anatomy, Faculty of Health Sciences, Aristotle University of Thessaloniki, Greece
}

[Received: 8 July 2018; Accepted: 29 July 2018]

\begin{abstract}
Neural and vascular variations in the axilla and upper limb area are usually paired, but coexistence of muscular aberration on top of this is uncommon. The current case report emphasizes on the unilateral coexistence of a three-headed (tricipital) biceps brachii muscle, a two-headed coracobrachialis with an accessory muscle bundle joining the superficial and deep heads of coracobrachialis muscle. On the ipsilateral side of the 72-year-old male cadaver, a connecting branch originated from the musculocutaneous nerve and joined the median nerve after surpassing the accessory muscle bundle. A large diameter subscapular trunk originated from the $2^{\text {nd }}$ part of the axillary artery and after giving off the $1^{\text {st }}$ lateral thoracic artery trifurcated into a common stem which gave off the $2^{\text {nd }}$ and $3^{\text {rd }}$ lateral thoracic arteries, the circumflex scapular artery and a common branch that gave off the $4^{\text {th }}$ and $5^{\text {th }}$ lateral thoracic arteries and the thoracodorsal artery, as the ultimate branch. All lateral thoracic arteries were accompanied by multiple intercostobrachial nerves. Documentation of such muscular and neurovascular variants and their embryologic origin increases awareness of their potential impact on diagnosis and treatment of upper limb pathology. To the best of our knowledge, the currently reported cadaveric observations seem to constitute a unique finding. (Folia Morphol 2019; 78, 2: 444-449)
\end{abstract}

Key words: biceps brachii, variation, coracobrachialis, lateral thoracic artery, intercostobrachial nerve, anastomosis, median nerve, musculocutaneous nerve

\section{INTRODUCTION}

The biceps brachii muscle (BB) is variable concerning the origin and insertion of its typical heads. In the majority of the reported cases, the long BB head arises from the superior glenoid labrum and the supraglenoid tubercle [1], while the short head arises from the apex of the coracoid process. The two heads join to form a single belly to be inserted to the radial tuberosity. Furthermore, BB is quite variable with regard to morphology, innervation and number (3-7) of accessory heads among different populations $[3,17,20]$. The existence of a $3^{\text {rd }} B B$ head is usually accompanied by axillary and brachial vessels with abnormal origin, branching pattern and course [15], variations in the musculocutaneous nerve (MCN) and coexistence of other multiple accessory muscles or

Address for correspondence: M. Piagkou, DDS, MD, MSc, PhD, Assistant Professor of Anatomy, School of Medicine, Faculty of Health Sciences, National and Kapodistrian University of Athens, 75 MikrasAsias Street, 11527 Athens, Greece, e-mail: mapian@med.uoa.gr 


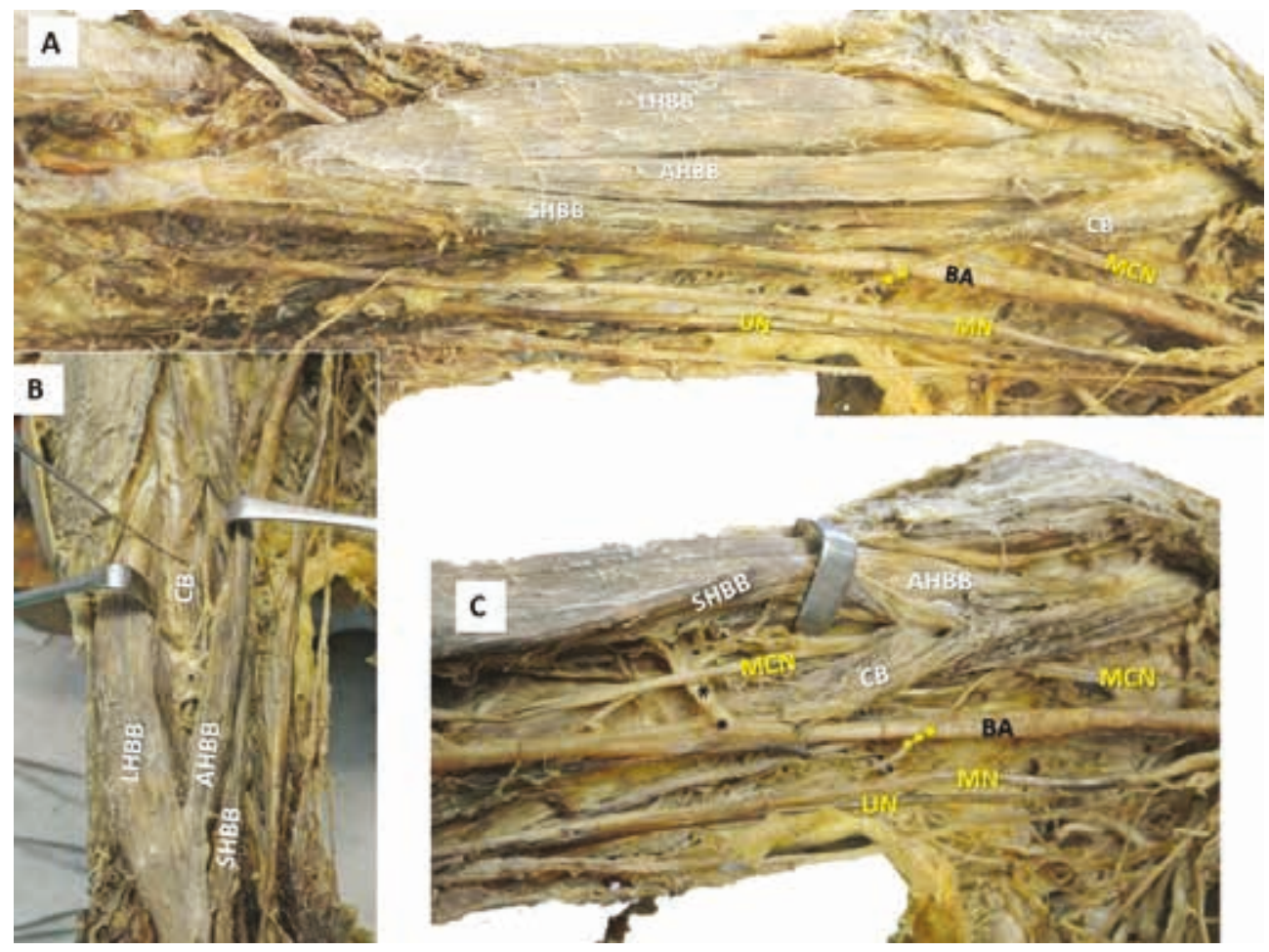

Figure 1. A. A three-headed biceps brachii muscle (BB) with the long, short and accessory heads (LHBB, SHBB and AHBB) combined with a two-headed coracobrachialis muscle (CB) with an accessory muscular bundle; $\mathbf{C}$. The musculocutaneous nerve (MCN) connecting branch $\left({ }^{* *}\right)$ initially coursed under the accessory muscular bundle, then advanced superficially to the brachial artery (BA) to ultimately reach and merge with the median nerve (MN); B. MCN innervated and BA supplied the atypical BB $\left({ }^{* *}\right)$; UN — ulnar nerve.

diversities of the coracobrachialis muscle (CB) [14]. Knowledge of anatomical variants in neurovascular and muscular structures is fundamental in interpreting imaging studies or unusual clinical manifestations.

The current case report emphasizes on the coexistence of a right-sided tricipital (three-headed) BB, a bicipital $C B$ which also bears an interconnecting accessory muscle bundle (AMB) joining the superficial with the deep head of the muscle. Neurovascular aberrations coexisted on the ipsilateral side of a 72-year-old Greek Caucasian male formalin fixed cadaver. Documentation of such muscular and neurovascular variants along with their embryologic origin explanation increases the awareness among clinicians regarding their potential impact on diagnosis and treatment of upper limb pathology. Complications during surgical exploration of the axilla and upper arm could be avoided by keeping in mind these variants. To the best of our knowledge, the reported observations, combined on a cadaver, appear to constitute a unique finding.

\section{CASE REPORT}

During the routine dissection of a 72-year-old formalin fixed Greek male cadaver, an unusual com- bination of muscular, neural and vascular variations was detected in the right axilla and upper arm. The cadaver was donated to the Department of Anatomy and Surgical Anatomy of the Medical School of the Aristotle University of Thessaloniki, through the "Anatomical Gift Programme" after a written informed consent was obtained. A three-headed BB was detected. The long head originated from the superior glenoid labrum and the supraglenoid tubercle similarly to Alashkham et al. study [1], the short head from the apex of the coracoid process and the accessory head from the tendon of the short head of $B B$ and $C B$. The accessory BB head after joining short and long heads inserted onto the posterior surface of the radial tuberosity. Innervation and vascular supply originated from the MCN and the brachial artery, respectively (Fig. 1). A bicipital (with superficial and deep heads) $C B$ coexisted with a connecting branch joining the MCN with the median nerve (MN). This branch originated from the MCN. An AMB extended between superficial and deep CB head. The MCN connecting branch surpassed the muscle bundle, on its way to the MN. The remaining MCN typically coursed between superficial and deep head of CB, supplying muscular branches to the anterior compart- 


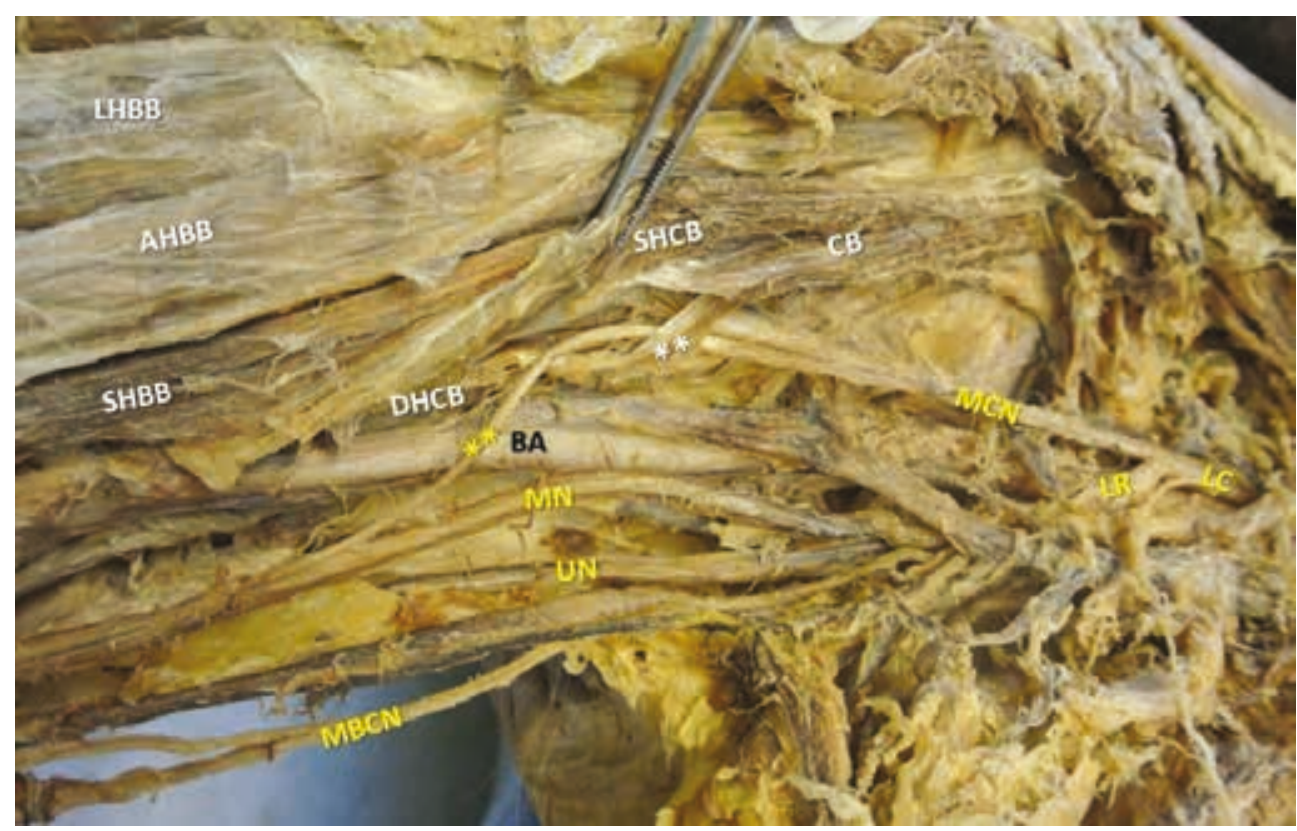

Figure 2. The tricipital biceps brachii muscle (BB) with the long, short and accessory heads (LHBB, SHBB and AHBB) combined with a bicipital coracobrachialis muscle (CB) (superficial and deep head SHCB and DHCB) with an accessory muscular bundle (white asterisks). Connecting branch (yellow asterisks) joining musculocutaneous nerve (MCN) with the median nerve (MN). MCN innervated and brachial artery (BA) supplied the atypical BB; UN — ulnar nerve; MBCN — medial brachial cutaneous nerve; LR — lateral root; LC — lateral cord.

ment of the arm (Fig. 2). The superior thoracic artery was absent and a large diameter subscapular arterial trunk (SST) originated just below the thoracoacromial trunk's origin ( $2^{\text {nd }}$ part of the axillary artery). The SST, just after the origin of the $1^{\text {st }}$ lateral thoracic artery (LTA), trifurcated into a common stem giving off the $2^{\text {nd }}$ and $3^{\text {rd }}$ LTA, the circumflex scapular artery (CSA) and a long branch that gave off the $4^{\text {th }}$ and $5^{\text {th }}$ LTA, and the thoracodorsal artery (TDA) as the ultimate branch. All LTAs were accompanied by multiple intercostobrachial nerves (ICBN). The $1^{\text {st }}$ LTA after its origin coursed anterior to the $1^{\text {st }} I C B N$ and the $2^{\text {nd }}$, $3^{\text {rd }}, 4^{\text {th }}$ and $5^{\text {th }}$ LTAs originated via a common trunk with the CSA and the TDA (Fig. 3). The left axilla and upper arm didn't show any aberration. The present study fully conforms to the provisions of the Helsinki Declaration of 1995 (as revised in Edinburgh 2000) and has been approved by the Ethics Committee of the Medical School of the Aristotle University of Thessaloniki, Greece.

\section{DISCUSSION}

Accessory heads of BB occur with an incidence ranging from $0.5 \%$ to $37.5 \%[8,12,20-22]$. Specifically, the incidence of the three-headed BB varies between $0.85 \%[14]$ and $20.5 \%[3,7]$ with the lowest prevalence in Europeans (0.85-10\%), Indians
(7.1\%), Chinese (8\%), Africans (12\%) and Turks (15\%) and the highest in Colombians (37.5\%), South Africans $(21.5 \%)$ and Japanese (18\%) $[3,6,8,9,14$, $21,24]$. Accessory heads, unilaterally or bilaterally found [13], may originate from the coracoid process and humeral shaft, the tendon or fascia of pectoralis minor or major, the deltoid muscle or CB insertion, the intermuscular septum, the greater tuberosity or the shoulder joint capsule [14, 23] and the anterior surface of brachialis muscle [18]. Although a male predominance was referred concerning the occurrence of the $3^{\text {rd }}$ head [2], no gender dimorphism exists $[8,9,13,22]$. Asymmetry has been reported by several authors [21] who predominantly reported a rightsided $3^{\text {rd }}$ head, similarly to the current case, while others mentioned left side supremacy $[2,5]$. Bilateral symmetrical accessory BB heads have an incidence ranging between $3.3 \%$ and $29.3 \%[3,9,13,21]$. All reported supernumerary BB heads are innervated by the MCN, with the rare exceptions where accessory heads are innervated by the $\mathrm{MN}[8,9,11,16]$.

In humans, where long head of $C B$ is absent, the third $B B$ head, which arises in continuity with $C B$ insertion, may represent a remnant of the $C B$ long head [22]. Muscles of the anterior arm compartment develop from myogenic precursor cells that arise from ventral dermomyotome of somites. Molecular chang- 


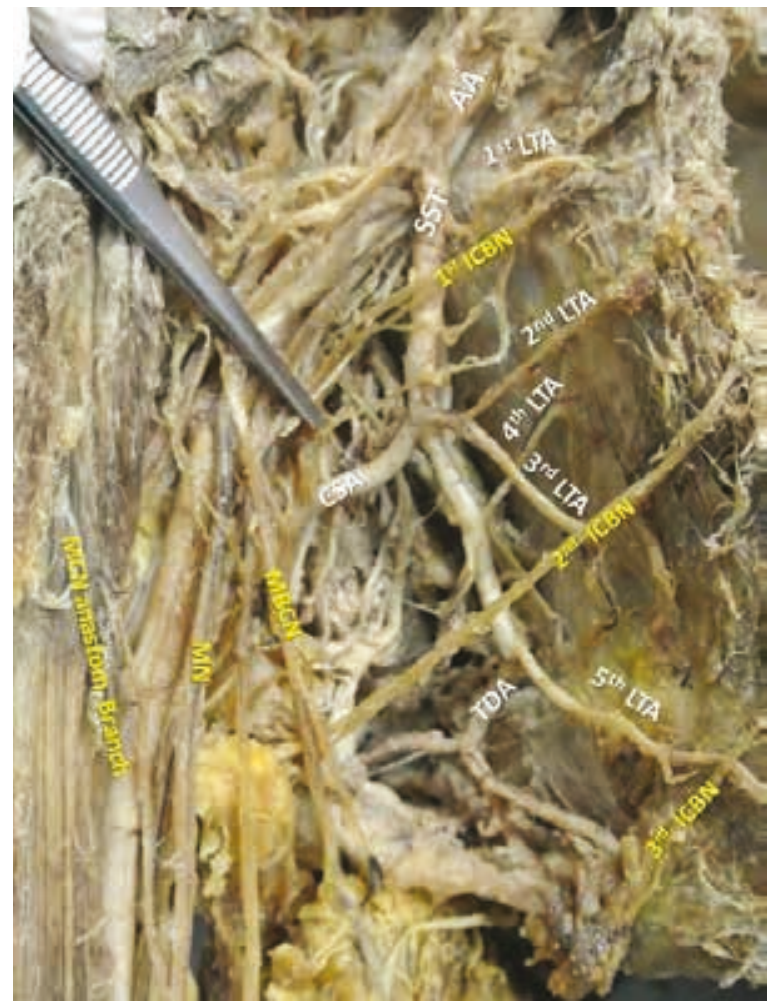

Figure 3. The subscapular arterial trunk (SST) coexisted with 5 lateral thoracic arteries (LTAs) and 3 intercostobrachial nerves (ICBNs). The $1^{\text {st }}$ LTA coursed anterior to the $1^{\text {st }}$ ICBN and the $2^{\text {nd }}$, $3^{\text {rd }}, 4^{\text {th }}$ and $5^{\text {th }}$ LTA originated via a common trunk with the circumflex scapular artery (CSA) and the thoracodorsal artery (TDA); $\mathrm{MCN}$ - musculocutaneous nerve; $\mathrm{MN}$ - median nerve; $\mathrm{MBCN}$ medial brachial cutaneous nerve.

es in these cells induce muscle development. Further growth of muscle occurs by fusion of myoblasts and myotubes. Variation of muscle patterns may be the product of altered signalling or stimulus between mesenchymal cells. A first hypothesis supports that the accessory BB heads may appear due to piercing of $B B$ by the MCN, thus resulting into a longitudinal splitting of myotubules, which eventually will be covered by a connective tissue layer producing a separate belly [11]. Developmental studies described the variation of the $3^{\text {rd }}$ BB head, as a portion of brachialis muscle supplied by the MCN, in which its distal insertion has been translocated from the ulna to the radius, according to the functional adaptation hypothesis. Circulatory factors during brachial plexus formation may affect the development of accessory heads [17]. Aberrant muscular formations may be remnants of muscle primordia that failed to disappear or fuse to form a sole structure [19]. According to embryological studies, it is possible that some of the fibres of the brachial plexus take an aberrant course, such as the connecting branch observed in the current study due to failure in differentiation [10].

Occasionally, the adjacent CB may have accessory muscle bundles inserting to the medial intermuscular septum, medial epicondyle, flexor carpi radialis, brachialis, brachioradialis or the pronator teres [13]. Course and direction of accessory heads or muscle bundles may occasionally entrap the $\mathrm{MN}$ and brachial artery $[16,19]$. The formation of a musculoaponeurotic bundle built up by fibres from $B B$ and $C B$, extending to the medial intermuscular septum and brachial fascia, may be a causative factor for forearm and hand neuro-vasculopathy [19]. MN entrapment and irritation may lead to tingling and neuropathic pain, followed or accompanied by reduced sensation or complete numbness. Compression of the brachial vessels may lead to ischaemia and oedema [19].

The accessory BB heads have been associated with MCN variations, such as an interconnecting branch with the MN [22], which was also found in our case. In such cases, the MCN course and branching pattern is usually affected and possible nerve entrapment may ensue. Rare exceptions of MN innervation have been also reported [16]. The MCN-MN interconnections are highly important to anaesthesiologists performing selective motor nerve blocks, to surgeons dealing with upper limb trauma management, as well as to clinicians and neurologists involved in nerve impairment treatment.

Presence of accessory BB heads and accompanying muscular aberrations may be combined with multiple neurovascular variations, as in the current case where multiple LTAs and ICBNs were detected. Five LTAs originated from the SST and the TDA. This rare variation is significant in clinical and surgical practice and was also referred by Loukas et al. [12] in $3.09 \%$. LTA variability was classified in types, in an effort to highlight the commonest (LTA from the thoracoacromial trunk, $67.62 \%$ ) and rarest (from the axillary $-17.02 \%$, the TDA - $5 \%$ and the subscapular artery $-3.93 \%$ ) variations. LTA point of origin is important, since the artery should remain intact during reconstructive procedures involving head and neck malignancies, modified radical mastectomy, mastopexy, breast reconstruction and reduction [12].

Concerning the ICBN variability, double, triple and multiple ICBNs with their possible anastomoses have been reported [10]. Usually ICBN is called the lateral cutaneous branch of the $2^{\text {nd }}$ intercostal nerve. 
A $2^{\text {nd }}$ ICBN often emanates from the anterior part of the $3^{\text {rd }}$ lateral cutaneous nerve supplying the axilla and the medial side of the arm. The ICBN may be preserved during radical mastectomy to prevent sensory loss or dysesthesia in the upper arm area [10]. Bratschi and Haller [4] emphasized on the significance of the ICBN, since a sensory loss or regional dysesthesia appears in approximately half of the performed breast cancer procedures and axillary lymphadenectomies (47.5\%). Less frequently, neural dysfunction may ensue when applying regional anaesthesia in upper limb procedures, with tourniquet compression, mechanical pressure therapy devices, entrapment and/or nerve traction [10].

From the clinical point of view, the supernumerary heads of BB usually remain asymptomatic, incidentally found during imaging studies or surgery. Radiologists should take into consideration the possibility of an accessory head and the accompanied neurovascular variations when assessing computed tomography and magnetic resonance imaging scans, as these features can be misinterpreted [24]. Clinicians should be also alert when dealing with patients complaining of weakness or pain in the anterior arm or when performing selective motor nerve blocks to treat nerve impairment cases [20]. Neural and vascular supply of the accessory heads is important to plastic and reconstructive surgeons [20]. Particularly, if the accessory head occurs unilaterally [6], it might be misinterpreted as a soft tissue tumour due to the asymmetry on palpation [16]. Accessory heads may also provide an additional force for supination and flexion of the forearm or they may cause unusual displacement of the humeral fragments if a fracture occurs. The medical records of the body donor don't mention any symptoms related to the underlying variations.

\section{CONCLUSIONS}

Accessory heads and muscular slips may alter anatomy of the arm, since they may be combined with unexpected multiple neural and vascular variations predisposing to complications due to their potential compressive action on nerves and superficially crossing brachial vessels. Clinicians should consider such anatomical variations in the differential diagnosis of patients presenting with ischaemia, oedema or MN palsy symptoms.

\section{Acknowledgements}

The authors express their thanks to the body donor and its family: without them this study would not have been possible.

\section{REFERENCES}

1. Alashkham A, Alraddadi A, Soames R. Origin of the long head of biceps brachii from the supraglenoid tubercle and glenoid labrum. Eur J Anat. 2018; 22: 213-219.

2. Ashraf Nasr $Y$, Adel Hussein M. Morphology and clinical implication of the extra-head of biceps brachii muscle. Folia Morphol. 2013; 72(4): 349-356, doi: 10.5603/ fm.2013.0058.

3. Asvat R, Candler P, Sarmiento EE. High incidence of the third head of biceps brachii in South African populations. J Anat. 1993; 182 (Pt 1): 101-104, indexed in Pubmed: 8509293.

4. Bratschi HU, Haller U. [Significance of the intercostobrachial nerve in axillary lymph node excision]. Geburtshilfe Frauenheilkd. 1990; 50(9): 689-693, doi: 10.1055/s-20081026346, indexed in Pubmed: 2272434.

5. Cheema P, Singla R. Lower incidence of the third head of biceps brachii in North Indian Population. J Clin Diagnostic Res. 2011; 5(Suppl.2): 1323-1326.

6. Kervancioglu P, Orhan M. An anatomical study on the three-headed biceps brachii in human foetuses, and clinical relevance. Folia Morphol. 2011; 70(2): 116-120, indexed in Pubmed: 21630233.

7. Kopuz C, Içten N, Yildirim M. A rare accessory coracobrachialis muscle: a review of the literature. Surg Radiol Anat. 2003; 24(6): 406-410, doi: 10.1007/s00276-002-0079-5, indexed in Pubmed: 12652369.

8. Kopuz C, Sancak B, Ozbenli S. On the incidence of third head of biceps brachii in Turkish neonates and adults. Kaibogaku Zasshi. 1999; 74(3): 301-305, indexed in Pubmed: 10429374.

9. Kosugi K, Shibata S, Yamashita H. Supernumerary head of biceps brachii and branching pattern of the musculocutaneus nerve in Japanese. Surg Radiol Anat. 1992; 14(2): 175-185, indexed in Pubmed: 1641744.

10. Kumar PA, Reddy DRK, Bapuji P. Multiple Intercostobrachial Nerves. J Evolution Med Dental Sciences. 2014; 57: 12978-12983.

11. Lokanadham S, Subhadra Devi V. Unusual presentation of supernumerary head of biceps brachii muscle in South Indian population. World J Med Sciences. 2011; 6: 115.

12. Loukas $M$, du Plessis $M$, Owens DG, et al. The lateral thoracic artery revisited. Surg Radiol Anat. 2013; 36(6): 543-549, doi: 10.1007/s00276-013-1234-x, indexed in Pubmed: 24281130.

13. Nakatani T, Tanaka S, Mizukami S. Bilateral four-headed biceps brachii muscles: the median nerve and brachial artery passing through a tunnel formed by a muscle slip from the accessory head. Clin Anat. 1998; 11(3): 209-212, doi: 10.1002/(SICI) 1098-2353(1998)11:3<209::AIDCA10>3.0.CO;2-N, indexed in Pubmed: 9579595.

14. Natsis K, Vlasis K, Tsakotos G, et al. case of a supernumerary third head of the biceps brachii muscle - clinical significance. Aristotle University Med J. 2010; 37: 39-41.

15. Natsis K, Piagkou M, Panagiotopoulos NA, et al. An unusual high bifurcation and variable branching of the axillary artery in a Greek male cadaver. Springerplus. 2014; 3: 640, doi: 10.1186/2193-1801-3-640, indexed in Pubmed: 25392808.

16. Nayak SR, Krishnamurthy A, Kumar MN, et al. Fourheaded biceps and triceps brachii muscles, with neurovascular variation. Anat Sci Int. 2008; 83(2): 107-111, 
doi: 10.1111/j.1447-073X.2007.00171.x, indexed in Pubmed: 18507620.

17. Nayak SR, Krishnamurthy A, Prabhu LV, et al. Multiple supernumerary muscles of the arm and its clinical significance. Bratisl Lek Listy. 2008; 109(2): 74-76, indexed in Pubmed: 18457314.

18. Ozan H, Atsev A. Sianau, A, Simsek, C, Basar R. An unusual insertion of the accessory biceps brachii muscle. Am Anat Nippon. 1997; 72: 515-519.

19. Paraskevas G, Natsis K, loannidis O, et al. Accessory muscles in the lower part of the anterior compartment of the arm that may entrap neurovascular elements. Clin Anat. 2008; 21(3): 246-251, doi: 10.1002/ca.20608, indexed in Pubmed: 18351653.

20. Rai R, Ranade AV, Prabhu LV, et al. Third head of biceps brachii in an Indian population. Singapore Med J. 2007; 48(10): 929-931, indexed in Pubmed: 17909679.
21. Rincón F, Rodríguez $Z$, Sánchez A, et al. The anatomic characteristics of the third head of biceps branchii muscle in a colombian population. Rev Chis Anat. 2002; 20(2): 197-200, doi: 10.4067/s0716-98682002000200014.

22. Rodríguez-Niedenführ M, Vázquez T, Choi $D$, et al. Supernumerary humeral heads of the biceps brachii muscle revisited. Clin Anat. 2003; 16(3): 197-203, doi: 10.1002/ ca.10060, indexed in Pubmed: 12673814.

23. Sargon MF, Tuncali D, Celik HH. An unusual origin for the accessory head of biceps brachii muscle. Clin Anat. 1996; 9(3): 160-162, doi: 10.1002/(SICI)10982353(1996)9:3<160::AID-CA4>3.0.CO;2-K, indexed in Pubmed: 8740475.

24. Vollala VR, Nagabhooshana S, Bhat SM, et al. Multiple arterial, neural and muscular variations in upper limb of a single cadaver. Rom J Morphol Embryol. 2009; 50(1): 129-135, indexed in Pubmed: 19221659. 